\title{
Eficiência bionutricional de animais da raça Nelore, F1s Valdostana-Nelore e de mestiços de raças européias adaptadas
}

[Bio-nutritional efficiency of Nellore, Valdostana-Nellore F1's and European adapted crossbred animals]

\author{
K. Euclides Filho' ${ }^{1}$, G.R. Figueiredo ${ }^{1}$, V.P.B. Euclides ${ }^{1}$, E.C. Vaz $^{2}$, J.B. Trovo ${ }^{1}$, \\ A.G. Razook ${ }^{3}$, L.A. Figueiredo ${ }^{3}$, L.O.C. Silva ${ }^{1}$, V. Rocco ${ }^{4}$ \\ ${ }^{1}$ Embrapa Gado de Corte \\ Caixa Postal 154 \\ 79002-970 - Campo Grande, MS \\ ${ }^{2}$ In memorian \\ ${ }^{3}$ Instituto de Zootecnia - Estação Experimental de Sertãozinho, Sertãozinho, SP \\ ${ }^{4}$ Bolsista de iniciação científica do $\mathrm{CNPq}$
}

\begin{abstract}
RESUMO
Foram avaliados cinco grupos genéticos quanto à eficiência bionutricional (índice resultante da primeira função canônica de uma análise bivariada que envolveu ganho de peso e consumo de matéria seca) sendo seis animais da raça Nelore $(\mathrm{N})$, cinco $1 / 2$ Romosinuano-1/2Caracu (RSC), quatro $1 / 2$ Senepol-1/2Caracu (SEC), quatro 1/2Belmont Red-1/2Caracu (BRC) e sete 1/2Valdostana-1/2Nelore (VAN). Não foi observada diferença significativa no desempenho dos animais mestiços Caracu, cuja média de eficiência bionutricional foi 519,8. O Nelore apresentou a menor eficiência bionutricional, 425,07, e os animais F1 Valdostana-Nelore, 499,30. Tendência semelhante foi observada para o ganho de peso. Os mestiços que envolveram raças européias adaptadas (RSC, SEC, BRC) apresentaram média de ganho de peso de $1,69 \mathrm{~kg} / \mathrm{dia}$, enquanto os F1s Valdostana-Nelore alcançaram $1,57 \mathrm{~kg} /$ dia e os da raça Nelore $1,29 \mathrm{~kg} / \mathrm{dia}$. Não houve diferença no consumo diário de matéria seca entre os grupos genéticos, média de $7,76 \mathrm{~kg}$ de MS ingerida/dia. Os grupos genéticos apresentaram conversão alimentar semelhante, com média de $4,87 \mathrm{~kg}$ de MS ingerida/kg de ganho de peso.
\end{abstract}

Palavras-chave: Nelore, Bos indicus, Bos taurus, consumo, conversão alimentar

\begin{abstract}
Five genetic groups were evaluated by bio-nutritional efficiency (index obtained from the first canonical function in a bivariate analysis encompassing weight gain and dry matter intake): six Nellore (N), five 1/2Romosinuano-1/2Caracu (RSC), four 1/2Senepol-1/2Caracu (SEC), four 1/2Belmont Red-1/2 Caracu $(B R C)$ and seven 1/2Valdostana-1/2Nellore (VAN). No differences were observed among the Caracu crosses for bio-nutritional efficiency, which averaged 519.8. Nellore showed the smallest performance (425.07), while the Vasdostana-Nellore F1's averaged 499.30. Similar trend was observed for daily weight gain. The crossbred animals involving European adapted breeds (RSC, SEC, BRC) showed an average gain of $1.69 \mathrm{~kg} /$ day, while the F1's Valdostana-Nelore and the Nellore gains were, respectively, $1.57 \mathrm{~kg} /$ day and $1.29 \mathrm{~kg} /$ day. No difference for dry matter (DM) intake among genetic groups which averaged $7.76 \mathrm{~kg}$ DM intake/day was observed. As far as feed conversion is concerned, no difference among genetic groups, which averaged $4.87 \mathrm{~kg}$ of DM intake $/ \mathrm{kg}$ of weight gain, was observed.
\end{abstract}

Keywords: Bos indicus, Bos taurus, feed conversion, intake

Recebido para publicação em 2 de julho de 2003

Recebido para publicação, após modificações, em 17 de março de 2004

E-mail: kepler@cnpgc.embrapa.br 


\section{INTRODUÇÃO}

A crescente demanda por alimentos livres de resíduos químicos, físicos e/ou biológicos fortalece a necessidade de se dedicar esforços na busca de animais resistentes e/ou tolerantes ao carrapato Boophilus microplus. Isto é, particularmente, importante para animais mestiços de zebu e europeu, por serem mais susceptíveis do que os zebuínos puros. O uso de animais tolerantes resulta, dentre outros benefícios, em manejo racional dos tratamentos químicos (Euclides Filho, 1999; 2000). Nessa ótica, aumenta a importância das raças zebus e das raças européias adaptadas para serem utilizadas tanto como raças puras, quanto em programas de cruzamentos.

Vários são os resultados que comprovam as diferenças entre os diversos cruzamentos envolvendo Bos taurus e Bos indicus, quer seja em termos de melhora da adaptabilidade, representada pela maior resistência aos parasitas internos e externos e à maior tolerância ao calor, quer seja ao incremento da produtividade (Morais, 1985a,b; Madalena et al., 1985; Oliveira e Alencar, 1986; Gomes et al., 1988; Euclides Filho et al., 2002).

Os cruzamentos que envolvem Bos indicus e Bos taurus não-adaptados com Bos taurus adaptados, as chamadas raças crioulas, têm resultado em melhora da produtividade e em carne mais macia, quando comparados com animais Bos indicus (Molina et al., 1982; Razook et al., 1986; Nardon et al., 1996; Chase et al., 1998).

Sob condições tropicais, a heterose, para características bioeconomicamente importantes, é normalmente oriunda de fatores determinantes básicos, quais sejam, potencial de produção com expressão plena sob condições ambientais favoráveis e capacidade de adaptação a condições estressantes representadas, principalmente, por resistência aos carrapatos e aos helmintos e pela tolerância ao calor. Essa argumentação, defendida por Frisch (1987), permite concluir que a produtividade dos bovinos em condições de estresse ambiental pode ser expressa como uma combinação entre a adaptabilidade e o potencial produtivo do animal. Assim, este trabalho objetivou avaliar o desempenho de animais cruzados envolvendo raças européias adaptadas, do Nelore e do cruzamento entre a raça Nelore e a raça Valdostana.

\section{MATERIAL E MÉTODOS}

Foram utilizadas informações obtidas de 26 animais, seis Nelore (N) e 20 F1 oriundos de cruzamentos, dos quais, 13 resultaram de cruzamentos entre raças européias adaptadas (quatro F1 Romosinuano $\times$ Caracu $(\mathrm{RSC})$, cinco F1 Senepol $\times$ Caracu (SEC) e quatro F1 Belmont Red $\times$ Caracu (BRC)), e sete eram F1 Valdostana $\times$ Nelore (VAN). Todos os animais foram produzidos em projetos em andamento na Embrapa Gado de Corte, em Campo Grande, MS. Os RSC, SEC e BRC pertenciam ao projeto "Composto Euro" e os VAN ao projeto "Embrapa Cruzamento 1". Foram todos criados em pastagens de Brachiaria decumbens, onde permaneceram com as mães até a desmama, que ocorreu, aproximadamente, aos seis meses de idade dos bezerros. Após a desmama, eles permaneceram em pastagens de Brachiaria brizantha por cerca de 30 dias sendo, em seguida, colocados em baia coletiva para adaptação ao novo manejo, por um período de 15 dias, onde receberam silagem de milho e concentrado. O concentrado, composto por $41 \%$ de milho triturado, $56 \%$ de farelo de soja, $2,98 \%$ de calcário calcítico e $0,02 \%$ de rumensin, foi fornecido de modo a representar $50 \%$ da matéria seca ofertada diariamente. Após esse período, foram colocados em baias individuais e passaram por um segundo período de adaptação de 15 dias, quando a quantidade de ração foi aumentada gradativamente até atingir o consumo estimado.

Durante o experimento, a quantidade de ração fornecida foi ajustada a cada três dias, possibilitando que as sobras ficassem em, aproximadamente, $10 \%$ da quantidade ofertada, como forma de garantir consumo ad libitum. A ração total foi distribuída duas vezes ao dia e tanto o fornecido como a sobra foram pesados diariamente. Todos os animais foram pesados mensalmente. Os animais permaneceram em avaliação até o abate, que ocorreu quando eles apresentavam, aproximadamente, de $3 \mathrm{~mm} \mathrm{a}$ $5 \mathrm{~mm}$ de gordura de cobertura. Esse grau de acabamento foi feito por avaliação visual por duas pessoas treinadas. 
Usou-se análise bivariada envolvendo o consumo e o ganho de peso dos animais, utilizando-se o procedimento MANOVA (User's..., 1989). As comparações foram realizadas considerando-se o índice obtido com base na primeira função canônica, definido por Euclides Filho et al. (2001) como eficiência bioeconômica. Foram utilizados quatro contrastes: $\mathrm{CC} 1$ - Nelore versus 1/2 Valdostana - 1/2 Nelore, CC2 - Nelore versus média de RSC, SEC e BRC, CC3 - média de RSC, SEC e BRC versus 1/2 Valdostana - 1/2 Nelore, e CC4 - SEC versus a média de RSC e BRC. Para as análises do ganho de peso diário médio, do consumo diário médio e da conversão alimentar média utilizou-se um modelo matemático que continha o efeito fixo de grupo genético e as covariáveis idade da vaca e idade do bezerro ao início do experimento. Esse modelo foi também usado para análise da eficiência bionutricional, definida como um índice resultante da primeira função canônica de uma análise bivariada, envolvendo ganho de peso e consumo de matéria seca.

\section{RESULTADOS E DISCUSSÃO}

As análises revelaram diferenças entre grupos genéticos para a eficência bionutricional e para o ganho de peso diário médio $(\mathrm{P}<0,05)$. Não foi observada diferença $(\mathrm{P}>0,05)$ para consumo diário médio de MS entre os grupos genéticos. As médias para essas três características encontram-se na Tab. 1. Em função desses resultados, somente foram avaliados contrastes para a eficiência bionutricional e para o ganho de peso diário médio. Esses contrastes encontram-se na Tab. 2.

Tabela 1. Médias de quadrados mínimos para eficiência bionutricional, ganho de peso e consumo de matéria seca de acordo com o grupo genético

\begin{tabular}{lccc}
\hline Grupo genético & Eficiência bionutricional & Ganho de peso (kg/dia) & Consumo de MS, kg/dia \\
\hline Nelore & 425,07 & 1,29 & 6,59 \\
$1 / 2$ Romosinuano - 1/2 Caracu & 561,46 & 1,68 & 7,94 \\
$1 / 2$ Senepol - 1/2 Caracu & 506,02 & 1,75 & 8,70 \\
$1 / 2$ Belmont Red - 1/2 Caracu & 512,24 & 1,64 & 7,84 \\
$1 / 2$ Valdostana - 1/2 Nelore & 499,30 & 1,57 & 7,74 \\
Média geral & 500,82 & 1,59 & 7,76 \\
\hline
\end{tabular}

Tabela 2. Contrastes entre médias de grupos genéticos para eficiência bionutricional, ganho de peso e consumo de matéria seca (MS)

\begin{tabular}{|c|c|c|c|}
\hline \multirow[t]{2}{*}{ Contraste $^{1}$} & $\begin{array}{c}\text { Eficiência } \\
\text { bionutricional }\end{array}$ & $\begin{array}{c}\text { Ganho de peso } \\
(\mathrm{kg} / \mathrm{dia})\end{array}$ & $\begin{array}{l}\text { Consumo de } \\
\text { MS, kg/dia }\end{array}$ \\
\hline & $\mathrm{P}>\mathrm{F}$ & $\mathrm{P}>\mathrm{F}$ & $\mathrm{P}>\mathrm{F}$ \\
\hline Nelore versus $1 / 2$ Valdostana - 1/2 Nelore & 0,0473 & $\mathrm{O}, 0439$ & 0,2202 \\
\hline Nelore versus a média de RSC, SEC e BRC & 0,0054 & 0,0135 & 0.1656 \\
\hline Média de RSC, SEC e BRC versus $1 / 2$ Valdostana - $1 / 2$ Nelore & 0,4294 & 0,4504 & 0,7910 \\
\hline SEC versus a média de RSC e BRC & 0,1487 & 0,9044 & 0,5287 \\
\hline
\end{tabular}

${ }^{1}$ RSC - 1/2 Romosinuano - 1/2 Caracu; SEC - 1/2 Senepol - 1/2 Caracu; BRC - 1/2 Belmont Red - 1/2 Caracu.

Observou-se melhor ganho de peso dos animais cruzados em relação aos da raça Nelore (1,66kg/dia versus $1,29 \mathrm{~kg} / \mathrm{dia})$. Apesar de não ter sido verificada diferença significativa de consumo entre os grupos genéticos, a diferença observada entre $\mathrm{N}$ e SEC foi superior a $30 \%$, o que pode representar variação prática relevante. Todavia, o número de animais utilizados pode ter sido insuficiente para fornecer maior poder ao teste.

Arq. Bras. Med. Vet. Zootec., v.56, n.5, p.671-675, 2004
Os animais Nelore apresentaram o pior desempenho $(\mathrm{P}<0,05)$. Entre os cruzamentos que envolveram as raças européias adaptadas não foi observada diferença quanto à eficiência bionutricional cuja média foi igual a 526,57. Também não houve diferença entre o desempenho desses animais e o observado para os mestiços Valdostana $(\mathrm{P}>0,44)$ cujo desempenho foi superior ao do Nelore. Essa diferença de desempenho nutricional entre Nelore e animais que envolvem cruzamentos de 
raças de origem européia foi também verificada por Euclides Filho et al. (1999), ao observarem melhor conversão alimentar dos animais cruzados.

Superioridade dos mestiços em relação ao Nelore para desempenho nutricional foi também observada por Euclides Filho et al. (2002). Esses autores, ao avaliarem o desempenho de animais Nelore e de cruzados 1/2Caracu-1/4Angus1/4Nelore (CCAN) e 1/2Caracu-1/4Simental$1 / 4$ Nelore (CCSN), verificaram que os cruzados apresentaram melhor eficiência bionutricional. Nesse estudo houve diferença entre o desempenho dos animais cruzados, isto é, os CCAN foram superiores aos CCSN. A literatura também registra resultados que não indicam diferenças quanto ao consumo de matéria seca entre grupos genéticos (Euclides Filho et al., 1997; Restle, et al., 2000). Perotto et al. (2002), ao estudarem animais das raças Canchim e Angus e seus cruzamentos recíprocos, observaram diferenças no consumo de MS. Os animais Canchim e seus filhos apresentaram o menor consumo. Tais diferenças foram também verificadas por Restle et al. (1995), ao avaliarem animais Charolês, Nelore e seus F1s recíprocos.

A análise da conversão alimentar segundo o grupo genético não resultou em diferenças significativas entre eles. As médias de conversão foram 5,$12 ; 4,79 ; 4,79 ; 4,70$ e 4,93 $\mathrm{kg}$ de MS ingerida $/ \mathrm{kg}$ de ganho de peso para os animais Nelore, 1/2Romosinuano-1/2Caracu, 1/2Senepol1/2Caracu, 1/2Belmont Red-1/2Caracu, e 1/2Valdostana-1/2Nelore, respectivamente. Estes resultados são semelhantes aos citados por Perotto et al. (2002) e Euclides Filho et al. (1997).

Os valores de conversão alimentar obtidos no presente trabalho sugerem excelente desempenho dos animais. Costa et al. (2002), ao trabalharem com novilhos Red Angus abatidos com quatro pesos finais, de $340 \mathrm{~kg}$ a $430 \mathrm{~kg}$, observaram valores de conversão entre $5,09 \mathrm{~kg}$ e $6,04 \mathrm{~kg}$ de $\mathrm{MS} / \mathrm{kg}$ de ganho de peso. Eifert (2000), citado por Costa et al. (2002), ao estudar animais Braford abatidos com $370 \mathrm{~kg}$ de peso vivo, verificou conversão alimentar igual a $4,34 \mathrm{~kg}$ de $\mathrm{MS} / \mathrm{kg}$ de ganho de peso. Dessa forma, estes resultados permitem antecipar que o uso desses cruzamentos pode contribuir para a produção de carne bovina em regiões tropicais e subtropicais.
Tal expectativa é ainda respaldada pelos resultados de Mujica et al. (1997) que, ao trabalharem com avaliação de cruzamentos envolvendo as raças Hereford e Senepol e seus F1, concluíram que há evidências de vantagens do uso de raças Bos taurus adaptadas (Senepol) em condições subtropicais. Baker et al. (2001), estudando o desempenho de cruzamentos envolvendo touros das raças Tuli, Senepol, Angus, Brahman e Polled Hereford com vacas "meio-sangue" Angus-Polled Hereford e Brahman-britânica, concluíram que as raças adaptadas avaliadas apresentam potencial para os sistemas de produção da faixa subtropical dos Estados Unidos.

\section{CONCLUSÕES}

Os cruzamentos que envolvem raças européias adaptadas podem se constituir em importantes componentes de sistemas de produção de bovinos de corte para as condições de Brasil Central Pecuário. Potencial semelhante foi expresso pelos animais F1s Valdostana-Nelore.

\section{AGRADECIMENTOS}

Os autores agradecem à Agropecuária CFM pela colaboração que tem tornado possível a condução desse projeto, especialmente pelo fornecimento de sêmen dos touros utilizados.

\section{REFERÊNCIAS BIBLIOGRÁFICAS}

BAKER, J.F; TUCKER, S.V.; VANN, R.C. Effects of Tuli, Senepol, Brahman, Angus, and Polled Hereford sire breeds on birth and weaning traits of offspring. Prof. Anim. Scient., v.17, p.160-165, 2001.

CHASE JUNIOR, C.C.; OLSON, T.A.; HAMMOND, A.C. et al. Preweaning growth traits for Senepol, Hereford, and reciprocal crossbred calves and feedlot performance and carcass characteristics of steers. $J$. Anim. Sci., v.76, p.2967-2975, 1998.

COSTA, E.C.; RESTLE, J.; PASCOAL, L.L. et al. Desempenho de novilhos Red Angus superprecoces, confinados e abatidos com diferentes pesos. Rev. Bras. Zootec., v.31, p.129-138, 2002.

EUCLIDES FILHO, K. Os cruzamentos na pecuária de corte brasileira. In: SIMPÓSIO INTERNACIONAL DE GENÉTICA E 
MELHORAMENTO ANIMAL, 1999, Viçosa. Anais... Viçosa: UFV:DZO, 1999. p.193-218.

EUCLIDES FILHO, K.; EUCLIDES, V.P.B.; FIGUEIREDO, G.R. et al. Avaliação de animais Nelore e seus mestiços com Charolês, Fleckvieh e Chianina, em três dietas. 1. Ganho de peso e conversão alimentar. Rev. Bras. Zootec., v.26, p.66-72, 1997.

EUCLIDES FILHO, K.; FIGUEIREDO, G.R.; EUCLIDES, V.P.B. et al. Desempenho de animais Nelore e seus mestiços com Caracu, Angus e Simental. In: REUNIÃO ANUAL DA SOCIEDADE BRASILEIRA DE ZOOTECNIA, 33., 1999, Porto Alegre. Anais... Porto Alegre: SBZ, 1999. p.

EUCLIDES FILHO, K.; FIGUEIREDO, G.R.; EUCLIDES, V.P.B. et al. Eficiência bionutricional de animais mestiços de raças européias e Nelore. Rev. Bras. Zootec., v.31, p.77-82, 2001.

EUCLIDES FILHO, K.; FIGUEIREDO, G.R.; EUCLIDES, V.P.B. et al. Eficiência bionutricional de animais nelore e seus mestiços com Simental e Aberdeen Angus, em duas dietas. Rev. Bras. Zootec., v.31. supl.1, p.331-334. 2002.

EUCLIDES FILHO, K.O composto de Bos taurus adaptado da Embrapa Gado de Corte. In: PECUÁRIA 2000. PERSPECTIVAS PARA O III MILÊNIO, 2000, Pirassununga. Anais... Pirassununga: FZEA-USP, junho de 2000. CD-ROM. Palestras.

FRISCH, J.E. Physiological reasons for heterosis in growth of Bos indicus x Bos taurus. J. Agric. Sci., v.109, p.213-230, 1987.

GOMES, A.; HONER, M.R.; SCHENK, M.A. et al. Populations of the cattle tick (Boophilus microplus) on purebred Nellore, Ibagé and Nellore x European crossbreds in the Brazilian savanna. Trop. Anim. Health Prod., v. 20, p. 124-130, 1988.

MADALENA, F.E.; TEODORO, R.L.; LEMOS, A.M. et al. Causes of variation of field burdens of cattle ticks (B. microplus). Rev. Bras. Gen., v.8, p.361-375, 1985.

MOLINA, R.S.; DEATON, O.W.; MUÑOZ, H. Potencial productivo del Romosinuano y sus cruzamientos para ganado de carne. Prod. Anim. Trop., v.7, p.272-277, 1982.

MORAIS, M. Tolerancia al calor de bovinos HolsteinCebú. II. Efecto de la temperatura ambiental y la humedad relativa en la temperatura rectal, frecuencia respiratoria y tasa sudoral en novillas cruzadas. Rev. Cub. Cienc. Vet., v.16, p.193-200, 1985a.
MORAIS, M. Tolerancia al calor de bovinos HolsteinCebú. III.Características del pelaje y peso corporal como criterio de adaptabilidad al clima cálido do húmedo de Cuba en hembras bovinas de los cruces $5 / 8$ $\mathrm{H}-3 / 8 \mathrm{C}$ (primer inter se), $5 / 8 \mathrm{H}-3 / 8 \mathrm{C} \mathrm{y} 3 / 4 \mathrm{H}-1 / 4$ C. Rev. Cub. Cienc. Vet., v.16, p.201-213, 1985 b.

MUJICA, F.; MENCHACA, M.A.; HAMMOND, A.C. et al. Comportamiento postdestete de novillos Senepol, Hereford y Senepol $\mathrm{x}$ Hereford, en Brooksville, Florida. Arch. Lat. Prod. Anim., v.5, p.155-166, 1997.

NARDON, R.; RAZZOK, A.G.; MOURA, A.C. et al. Influência da raça, época de abate e metodologia de análise nas características quali-quantitativas de carcaças de bovinos selecionados para ganho de peso. In: REUNIÃO ANUAL DA SOCIEDADE BRASILEIRA DE ZOOTECNIA, 33., 1996, Fortaleza. Anais ... Fortaleza: SBZ, 1996. p.200-202.

OLIVEIRA, G. P.; ALENCAR, M. M. Resistência de bovinos Canchim ao carrapato (Boophilus microplus). In: REUNIÃO ANUAL DA SOCIEDADE BRASILEIRA DE ZOOTECNIA, 25., 1986, Campo Grande. Anais... Campo Grande: SBZ, 1986. p.262.

PEROTTO, D., MOLETTA, J.L., LESSKIU, C. Desempenho em confinamento de machos bovinos inteiros Canchim, Aberdeen Angus e cruzamentos recíprocos. Ciênc. Rural, v.32, p.669-674, 2002.

RAZOOK, A.G.; LEME, P.R.; PACKER, I.U. et al Evaluation of Nellore, Canchim, Santa Gertrudis, Holstein, Brown Swiss and Caracu as sire breeds in matings with Nellore cows. I. Effects on progeny growth, carcass traits and crossbred productivity. In: WORLD CONGRESS ON GENETICS APPLIED TO LIVESTOCK PRODUCTION, 3., 1986, Lincoln. Proceedings... Lincoln: University of Nebraska Board of Regents, 1986. p.348-352.

RESTLE, J.; FELTEN, H.G.; VAZ, F.N. Efeito da raça e heterose para desempenho em confinamento de novilhos de corte. In: REUNIÓN LATINOAMERICANA DE PRODUCCIÓN ANIMAL, 14. 1995, Mar del Plata. Memorias... Balcarce:ALPA, 1995. p.852-854.

RESTLE, J.; QUADROS, A.R.B.; VAZ, F.N. Terminação em confinamento de" novilhos de diferentes genótipos Hereford x Nelore. Rev. Bras. Zootec., v.29, p.125-130, 2000.

USER's guide: Statistics version 6. 4.ed. Cary, NC: SAS Institute, 1989. V.1, 943p. 